\title{
A Schadenfreude Inducing Misfortune Reduces Envy
}

\author{
Niels van de Ven - Tilburg University
}

Three studies find that when a schadenfreude eliciting misfortune happens to an envied person, this reduces subsequent envy. The results are consistent with the idea that schadenfreude serves as a signal that the prior imbalance in how one would like the world to see (caused by the other being better off than we would like him/her to be) is restored again.

Acknowledgments: The author thanks Tim Otten with his help in conducting Study 3. 
In the current set of studies we test the hypothesis that when an envied person suffers a minor misfortune, this triggers schadenfreude, after which the initial envy is reduced. We base this on the idea of Van de Ven (2018), that schadenfreude is best understood as a signal that things are well again (or better at least). An antecedent of schadenfreude is that there is a violation of how we would want the world to be: someone else whom we do not look positively upon has a position that we do not think (s)he should be in (see Smith \& Van Dijk 2018). If this person then suffers a misfortune, the positive feeling of schadenfreude signals to us that the balance is restored and the world is again closer to as we would want it to be. Envy is the frustrating feeling that someone else is better off than we are (Smith \& Kim, 2007; Van de Ven, 2016; Lange, Weidman, \& Crusius, 2018). As envy arises over a specific form of an imbalance (the other having something that we want or do not want him/her to have, Van de Ven, Zeelenberg, \& Pieters, 2009), a misfortune happening to them might help to restore some of that balance. People do indeed tend to feel more schadenfreude when a (maliciously) envied person suffers a misfortune (Van de Ven et al., 2015). But does experiencing schadenfreude reduce subsequent envy?

In the current set of studies we test whether a schadenfreude inducing misfortune reduces existing envy. Note that the misfortune in our studies does not alter the actual envy-eliciting situation, and that the envied person still has the advantage that triggered the envy in the first place. Still, we expect that schadenfreude is interpreted as a signal that something happened that restored part of the imbalance, providing a signal that envy should become a less important emotion. The idea is that this can be functional, as when the situation becomes less important (e.g., when an envied person loses some of his or her status) people can refocus on other things that become relatively more important again (see the broaden-and-build model of emotions, 
Fredrickson, 2001). ${ }^{1}$ We conduct three studies to test this idea. The first two use hypothetical situations, the third one an ostensibly real one.

\section{Study 1}

Participants imagined being in a situation designed to elicit envy, after which they read of a misfortune that befell the envied other. In one condition, participants indicated how envious they would be, after which the misfortune occurred and they indicated how much schadenfreude they would experience. After this we again measured how envious they would be at that time. This allowed us to test within-subjects whether envy after a misfortune that elicits schadenfreude would be lower than before the misfortune.

We also added a second condition, in which participants did not start by answering questions on envy. In this condition they first read about both the situation that elicits envy, then about the misfortune, and only then answered the questions on schadenfreude and envy. This allowed us to test 1) whether self-reported schadenfreude is not influenced by asking them about envy first and 2) also conduct a between-subjects test on whether envy before the misfortune is higher than experienced envy after a misfortune.

\section{Method}

Participants. Participants were recruited via Amazon Mechanical Turk and were paid \$0.20 for a short study. We aimed for 160 participants, divided over two conditions. The main test is a within-subject test in one of the conditions, comparing the envy before the misfortune to the envy after the misfortune, and 80 participants in this condition would give almost $90 \%$ power

\footnotetext{
${ }^{1}$ Note that this manuscript is intended as a sort of "data-dump", where I present some findings that at the moment of writing this manuscript are unlikely to be published elsewhere. As they might be of interest to others, I wanted to make the data available as well. The idea of schadenfreude as a signal of restored balance is explained in Van de Ven (2018).
} 
to detect a $d=0.40$. We ended up with 161 participants who completed the study, 107 male, 54 female, $M_{\mathrm{age}}=30.27, \mathrm{SD}=7.92$, range $19-63$.

Procedure. Participants were asked to imagine being in the following situation, and to write down a few words that came to their mind when thinking about how they would feel in that situation (to stimulate them to mentally place themselves in the situation):

You have been putting a lot of effort into your work lately and performed well. Colleagues told you that your manager is considering you for a promotion. This would be very welcome, as you feel you are ready for the next step in your career. At a meeting, your manager announces that your colleague P. gets the promotion. You feel that this is rather undeserved, as you think you've done a better job than P did.

After this they indicated how much envy they felt on three questions (would you feel a little envious of P? / would you feel jealous of P? / would you feel frustrated that $\mathrm{P}$ had the promotion and you did not?, $\alpha=.79)$. All questions in this manuscript were always measured on a scale from 0 (not at all) to 6 (very much so). These envy questions were the same as those used by Van de Ven (2017) to measure general envy, adjusted to this situation by referring to the colleague $\mathrm{P}$ and the promotion.

After this they were asked to imagine the following:

Now imagine that the person who had received the promotion, P., makes a serious mistake at work. Due to carelessness of $\mathrm{P}$, a large customer cancels their order which reduces the profit of your organization. Your manager is not happy with this.

They then indicated how they felt by responding to three questions that tapped into their experienced schadenfreude (I would be a little amused by what happened to him/her / I would secretly be pleased by the misfortune that happened to him / her, I'd find it difficult to resist a little smile, $\alpha=.92$ ). These schadenfreude questions are the same as used by Van de Ven et al (2015). 
After this, we again measured their envy towards their colleague with the exact same questions as before. The reliability of this measure was lower, with $\alpha=.66$. This relatively low alpha mainly seemed to be the case because one question (would you feel frustrated that $\mathrm{P}$ had the promotion and you did not?) fitted less well with the other two questions on envy and jealousy this time. Excluding this question does not change any of our results, we therefore chose to keep it in to keep the measure consistent with earlier work.

The procedure as we described it so far is the procedure for all participants who were in the Pre- and Post-Schadenfreude Envy condition $(\mathrm{N}=78)$. The other participants $(\mathrm{N}=83)$ followed the exact same procedure, but did not fill out the envy measure before the schadenfreude episode (Post-Schadenfreude Envy Only condition).

\section{Results}

Descriptive statistics for all variables can be found in Table 1. As expected, there was no difference between the conditions in the experienced schadenfreude, $t(159)=0.67, p=.502, d=$ 0.11 , or the envy towards the other person after the misfortune happened, $t(159)=1.18, p=.239$, $d=0.19$. This implies that asking for envy before the misfortune did not affect the ratings of schadenfreude or envy after the misfortune.

Past research found that when people are (maliciously) envious of someone, they typically experience more schadenfreude when that person suffers a misfortune (Van de Ven et al., 2015). Our current hypothesis is that after experiencing schadenfreude in such situations, envy will be reduced. For participants who had indicated their envy towards the other both before and after the misfortune, we indeed find that the envy after the schadenfreude-inducing misfortune was lower (see Table 1), paired-t(77) $=14.11, p<.001, d_{z}=1.60$. When we perform a between-subjects test, comparing the envy of participants who rated it immediately after the 
envy eliciting story, to envy experienced after the misfortune by the participants who only rated envy at that time, we find the same effect, $t(159)=10.39, p<.001, d=1.63 .^{2}$ To summarize, we find that after a misfortune that triggers schadenfreude, envy is indeed reduced.

\section{Study 2}

Study 2 was designed to replicate and extend the findings of Study 1. First, a main goal was to replicate the finding that envy is reduced after a misfortune. A second goal was to explore the effects of schadenfreude on the two subtypes of envy. Both are frustrating experiences when someone else is better off than oneself, but benign envy activates a motivation to improve oneself, while malicious envy activates a motivation to pull down the other person (Van de Ven, Zeelenberg, \& Pieters, 2009). We measured both these motivations that are part of these envy subtypes, to see whether the schadenfreude would reduce one or both of these envy types. ${ }^{3}$

\section{Method}

Participants. Participants were recruited via Amazon Mechanical Turk and were paid $\$ 0.30$ for a short study. We aimed for 600 participants, randomly divided over one of four

\footnotetext{
${ }^{2}$ I considered whether I expected that more intense schadenfreude would be related to a greater reduction in envy. After all, if schadenfreude is something akin to a signal that for example envy is no longer needed, more intense schadenfreude might be expected to lead to a stronger drop in envy. Another expectation is that the same misfortune suffered by an envied person should lead to similar drops in envy for everyone, regardless of their prior envy (as it is the same misfortune after all). If this is true, this would lead to a different prediction: as the same misfortune leads to similar drops in envy for everyone, but schadenfreude is more intense for those with more prior envy (see Van de Ven et al., 2015), schadenfreude might still be positively related to envy after the misfortune as well. To summarize, we did not know what to expect here. The Appendix contains explorative tests for the effects of intensity of schadenfreude on envy in two ways: schadenfreude predicting the difference between envy pre- and postmisfortune (Appendix Table 1), and as part of a regression in which envy after the misfortune is predicted by both prior envy and schadenfreude (appendix Table 2).

${ }^{3} \mathrm{~A}$ third goal was to further explore if there are boundary conditions to the reducing effect of schadenfreude on envy. To do so, we added two manipulations. First, we manipulated whether the advantage held by the other was undeserved or deserved (Study 1 only used an undeserved advantage). The second manipulation was that we manipulated whether the misfortune was in a domain related to the domain in which the other person was better off, or in a different domain than the one in which the other was better off to explore whether this matters for the envy reduction.
} 
conditions of a 2 (Advantage of Other: Undeserved versus Deserved) x 2 (Domain of Misfortune: Same versus Different). This sample size allowed for well-powered tests, also to explore possible correlations between schadenfreude and envy. We ended up with 602 participants who completed the study, 342 male, 260 female, $\mathrm{M}_{\mathrm{age}}=33.81, \mathrm{SD}=10.70$, range 18-67. There were 139 participants in the Undeserved / Same Domain condition, 158 in the Undeserved / Different Domain condition, 157 in the Deserved / Same Domain condition, and 148 in the Deserved / Different Domain condition.

Procedure. The procedure was in general the same as in Study 1. Participants were asked to imagine being in the situation in which a colleague got a promotion that they had wanted themselves, and to write down a few words that came to their mind when thinking about how they would feel in that situation. The Undeserved condition was practically the same as in Study 1 , in the Deserved condition the sentence on the undeservedness of the situation was replaced by the one in brackets:

You have been putting a lot of effort into your work lately and performed well. Colleagues told you that your manager is considering you for a promotion. This would be very welcome, as you feel you are ready for the next step in your career.

At a meeting, your manager announces that your colleague P. gets the promotion. You feel that this is actually rather undeserved, as you think you've done a better job than $P$ did. [You feel that this is actually rather deserved, as you think $P$ did a better job than you did so far.] You feel upset that you did not manage to get the promotion.

Participants then indicated how much envy they felt on same three questions as in Study 1 ( $\alpha=$ .86). After this they received four questions tapping into the motivation following benign envy ( $\alpha$ $=.72$, an example is "I would feel inspired to get the promotion myself") and four tapping into the motivation following malicious envy ( $\alpha=.95$, an example item is "I would want P to lose the promotion"), with the order of these two sets of questions randomized. 
After these questions they again read about a misfortune happening to their colleague $\mathrm{P}$. They either read the misfortune also used in Study 1 (due to carelessness of colleague P, a large customer cancels an order), which was in the same domain as the one in which colleague P was better off than the participant by having received the promotion. In another condition participants read about a misfortune unrelated to the domain in which the other was better off:

Now imagine that the person who had received the promotion, P., makes an error while parking a car in front of a store. Many people were at the parking lot and saw it happen. P's car hits another person's car, leaving a large dent in both cars. The other person is not happy with this.

This misfortune is unrelated to colleague $\mathrm{P}$ having the promotion, and is therefore unrelated to the domain of envy. After reading about these misfortunes, participants responded to the same three schadenfreude questions as before $(\alpha=.95)$. They then again indicated their felt envy $(\alpha=.91)$, felt motivation to improve $(\alpha=.87)$, and felt motivation to pull down the other $(\alpha$ $=.95)$, of which the latter two sets of questions were randomized.

\section{Results}

We first analyzed the results when combining all conditions, as a replication of the within-subject effects of Study 1. We then explore the within-subject effects on the motivations associated with benign and malicious envy, after which we explore the effects of the manipulations of deservedness and domain of the misfortune. Descriptive statistics for all variables can be found in Table 2 .

Reduction of envy following the misfortune. Our main hypothesis is that when people are envious of someone, they become less envious after the envied person suffered a misfortune. We again found that the envy after the misfortune was lower than before the misfortune, paired$t(601)=18.11, p<.001, d_{z}=0.74$ 


\section{Reduction of envy's action tendencies following the misfortune. We performed}

similar tests on the motivations that typically follow benign and malicious envy, to see whether schadenfreude also reduced one or both of these. First, for the motivation to move up when the envied person was better off, we also found that this motivation was somewhat reduced after the misfortune happened to the other (from $M=4.70, S D=1.04$, to $M=4.60, S D=1.24$ ), paired$t(598)=3.14, p=.002, d_{z}=0.13$. This was also the case for the motivation to pull down the other (from $M=2.13, S D=1.84$, to $M=1.95, S D=1.82$ ), paired- $t(598)=4.44, p<.001, d_{z}=$ 0.18. Although the effects are clearly small, when a misfortune happens to an envied person it does appear to reduce the action tendencies associated with both benign and malicious envy.

Effects of manipulations of similarity and deservingness. The reduction in envy following the misfortune was stronger when the domain of the misfortune was similar to the domain in which the other held an advantage, $F(1,598)=5.68, p=.017, \mathrm{\eta}_{\mathrm{p}}^{2}=.01$, and when the advantage had been undeserved, $F(1,598)=7.27, p=.007, \eta_{\mathrm{p}}{ }^{2}=.01$.

To summarize, Study 2 again found that when an envied person suffers a schadenfreude inducing misfortune, the resulting envy is lower. This effect occurs in situations in which the misfortune happens in another or the same domain of the envy (but is stronger in the latter case), and in situations in which the prior advantage held by the envied person was deserved or undeserved (but is stronger in the latter case).

\section{Study 3}

In Study 3 we aimed to replicate these effects in a setting where we tried to induce actual envy and schadenfreude, instead of the hypothetical situations used in Studies 1 and 2. Participants read a success story of another mTurk worker (adapted from Lange and Crusius, 
2015), after which we measured their envy. They then read about a misfortune happening to this person in another context, and we measured schadenfreude and envy again.

\section{Method}

Participants. Participants were recruited via Amazon Mechanical Turk and were paid $\$ 0.48$ for a short 4 minute study. We aimed for 600 participants. We ended up with 567 participants who completed the study, 318 male, 222 female, 27 information missing, $\mathrm{M}_{\mathrm{age}}=$ $35.83, \mathrm{SD}=10.33$, range $19-67$.

Procedure. Participants were asked to form an impression of another mTurk worker (Alex), who was quite successful on mTurk (see Lange \& Crusius, 2015). The mTurk worker had the coveted Master worker and earned a good hourly wage. We asked participants how envious they were of Alex with the three items used before $(\alpha=.83)$. Next, they read about a misfortune occurring to Alex when dating (a different domain compared to the envy-eliciting situation). Alex wrote that on a date he sneezed while eating spaghetti, creating a mess. This made people at surrounding tables laugh, but not his date. We again measured schadenfreude using the items used before $(\alpha=.73)$ and measured envy again $(\alpha=.83){ }^{4}$

Results. Participants felt some schadenfreude towards the misfortune, $M=2.14, \mathrm{SD}=$ 1.46). The envy before the misfortune $(M=2.98, \mathrm{SD}=1.70)$ was again higher than the envy after the misfortune $(M=2.51, \mathrm{SD}=1.74)$, paired- $t(566)=12.30, p<.001, d_{z}=0.52$.

\section{General Discussion}

Why do people experience schadenfreude? There are several possible reasons for this (see Smith, Powell, Combs, \& Schurtz, 2009), but one additional reason could be that schadenfreude is a signal that the world is again (a bit closer to) what we want it to be (see Van

\footnotetext{
${ }^{4}$ Note that we also measured the moving up motivation associated with benign envy and the pulling down motivation associated with malicious envy both before and after the misfortune.
} 
de Ven, 2018, for a more thorough explanation). The current results we find in three studies, that envy is reduced after a schadenfreude eliciting misfortune that happened to the envied person, is consistent with this idea. 


\section{References}

Fredrickson, B. L. (2001). The role of positive emotions in positive psychology: The broadenand-build theory of positive emotions. American Psychologist, 56, 218-226. doi:10.1037/0003-066X.56.3.218

Lange, J., \& Crusius, J. (2015). Dispositional envy revisited: Unravelling the motivational dynamics of benign and malicious envy. Personality and Social Psychology Bulletin, 41, 284294. doi:10.1177/0146167214564959

Lange, J., Weidman, A.C., \& Crusius, J. (2018). The painful duality of envy: Evidence for an integrative theory and a meta-analysis on the relation of envy and schadenfreude. Journal of Personality and Social Psychology. doi:10.1037/pspi0000118

Smith, R. H., \& Kim, S. H. (2007). Comprehending envy. Psychological Bulletin, 133, 46-64. doi:10.1037/0033-2909.133.1.46

Smith, R. H., Powell, C. A. J., Combs, D. Y., \& Schurtz, D. R. (2009). Exploring the when and why of schadenfreude. Social and Personality Psychology Compass, 3, 530-546. doi:10.1111/j.1751-9004.2009.00181.x

Smith, R. H., \& Van Dijk, W. W. (2018). Schadenfreude and Gluckschmerz. Emotion Review. Van de Ven, N. (2016). Envy and its consequences: Why it is useful to distinguish between benign and malicious envy. Social and Personality Psychology Compass, 106, 337-349. doi:10.1111/spc3.12253

Van de Ven, N. (2017). Envy and admiration. Emotion and motivation following upward social comparison. Cognition and Emotion, 31(1), 193-200. doi:10.1080/02699931.2015.1087972

Van de Ven, N. (in press). Schadenfreude and Gluckschmerz Are Emotional Signals of (Im)Balance. Emotion Review. 
Van de Ven, N., Hoogland, C., Smith, R. H., Van Dijk, W. W., Breugelmans, S. M., \& Zeelenberg, M. (2015). When envy leads to schadenfreude. Cognition and Emotion, 29(6), 1007-1025. doi:10.1080/02699931.2014.961903

Van de Ven, N., Zeelenberg, M., \& Pieters, R. (2009). Leveling up and down: The experience of benign and malicious envy. Emotion, 9, 419-429. doi: 10.1037/a0015669 
Table 1.

\begin{tabular}{lcccc}
\hline & \multicolumn{2}{r}{ Pre- and Post-Schadenfreude Envy } & \multicolumn{2}{c}{ Post-Schadenfreude Envy Only } \\
& $\mathrm{M}$ & $\mathrm{N}=78$ & $\mathrm{~N}=83$ & (SD) \\
& 4.27 & $(1.39)$ & - & - \\
\hline Envy before misfortune & 3.49 & $(2.01)$ & 3.69 & $(1.60)$ \\
Schadenfreude & 2.03 & $(1.28)$ & 2.25 & $(1.06)$ \\
Envy after misfortune & & & & \\
\hline
\end{tabular}


Table 2.

\begin{tabular}{|c|c|c|c|c|c|c|c|c|c|c|}
\hline & & & \multicolumn{4}{|c|}{ Undeserved } & \multicolumn{4}{|c|}{ Deserved } \\
\hline & \multicolumn{2}{|c|}{ Total } & \multicolumn{2}{|c|}{ Same Domain } & \multicolumn{2}{|c|}{ Different Domain } & \multicolumn{2}{|c|}{ Same Domain } & \multicolumn{2}{|c|}{ Different Domain } \\
\hline & M & $(\mathrm{SD})$ & M & $(\mathrm{SD})$ & M & $(\mathrm{SD})$ & M & $(\mathrm{SD})$ & M & $(\mathrm{SD})$ \\
\hline Envy before Misfortune & 3.59 & $(1.65)$ & 4.00 & $(1.53)$ & 4.02 & $(1.57)$ & 3.19 & $(1.59)$ & 3.19 & $(1.71)$ \\
\hline Move Up before Misfortune & 4.70 & $(1.04)$ & 4.65 & $(0.94)$ & 4.57 & $(1.11)$ & 4.74 & $(1.01)$ & 4.86 & $(1.06)$ \\
\hline Pull Down before Misfortune & 2.13 & $(1.84)$ & 2.96 & $(1.81)$ & 2.94 & $(1.76)$ & 1.25 & $(1.44)$ & 1.42 & $(1.63)$ \\
\hline Schadenfreude & 2.21 & $(1.94)$ & 2.95 & $(1.95)$ & 2.66 & $(2.09)$ & 1.68 & $(1.62)$ & 1.61 & $(1.71)$ \\
\hline Envy after Misfortune & 2.46 & $(1.77)$ & 2.51 & $(1.70)$ & 2.90 & $(1.81)$ & 2.11 & $(1.67)$ & 2.33 & $(1.81)$ \\
\hline Move Up after Misfortune & 4.60 & $(1.24)$ & 4.54 & $(1.22)$ & 4.49 & $(1.31)$ & 4.59 & $(1.19)$ & 4.78 & $(1.21)$ \\
\hline Pull Down after Misfortune & 1.95 & $(1.82)$ & 2.58 & $(1.92)$ & 2.54 & $(1.80)$ & 1.27 & $(1.47)$ & 1.47 & $(1.71)$ \\
\hline
\end{tabular}


Appendix Table 1. Schadenfreude as predictor of the difference in envy pre- to post misfortune

\begin{tabular}{cccc}
\hline & $\mathrm{N}$ & $\beta$ & $p$ \\
\hline Study 1 & 78 & -.22 & .055 \\
Study 2 & 602 & -.12 & .004 \\
Study 3 & 567 & .09 & .035
\end{tabular}

Note. A positive $\beta$ implies that an increase in schadenfreude is related to a smaller differences pre- and post-misfortune, a negative $\beta$ implies that an increase in schadenfreude is related to a greater reduction in envy following a misfortune.

Appendix Table 2. Schadenfreude and envy pre-misfortune as predictors of envy post-misfortune

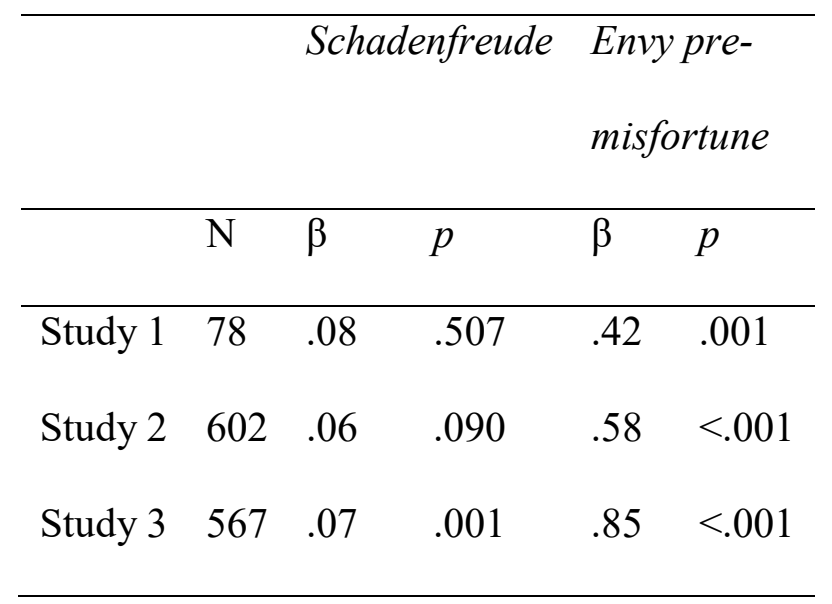

